Itinéraires Itinéraires

Littérature, textes, cultures

\title{
Laura Doyle and Laura Winkiel (dir.), Geomodernisms. Race, Modernism, Modernity
}

Nathalie Carré

\section{OpenEdition}

Journals

Édition électronique

URL : http://journals.openedition.org/itineraires/568

DOI : 10.4000/itineraires.568

ISSN : 2427-920X

Éditeur

Pléiade

Édition imprimée

Date de publication : 1 novembre 2009

Pagination : 190-192

ISBN : 978-2-296-10115-9

ISSN : 2100-1340

\section{Référence électronique}

Nathalie Carré, "Laura Doyle and Laura Winkiel (dir.), Geomodernisms. Race, Modernism, Modernity», Itinéraires [En ligne], 2009-3 | 2009, mis en ligne le 27 juin 2014, consulté le 22 septembre 2020. URL http://journals.openedition.org/itineraires/568 ; DOI : https://doi.org/10.4000/itineraires.568

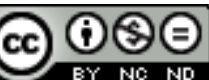

Itinéraires est mis à disposition selon les termes de la licence Creative Commons Attribution - Pas d'Utilisation Commerciale - Pas de Modification 4.0 International. 
Ainsi, sous le titre ambitieux, L'Avant-Garde européenne, dans une collection explicitement consacrée à l'histoire culturelle de la littérature, ce livre rassemble surtout une vaste collection d'explications de textes, d'interprétations de tableaux ou de séquences filmées, reliées entre elles par une intarissable fièvre théorique. Webber ne s'intéresse ni aux conditions de productions, ni au contexte historique, ni aux carrières, ni aux transferts culturels entre avant-gardes, mais seulement à des fragments extraits de tel ou tel artiste dit «d'avant-garde ». Son intérêt se porte avant tout sur la théorie, il recourt à des termes fortement chargés de sens, tels que le matérialisme, la psyché, l'inconscient, mais en fait un usage si extensif qu'on finit un peu perdu entre une métaphore filée à visée rhétorique, et un jeu de mot faisant office de transition entre les éléments de son corpus.

D'une lecture suivie très exigeante, l'ouvrage saura peut-être retenir l'attention de lecteurs intéressés par l'application des théories freudiennes aux productions littéraires et artistiques, et éveiller l'intérêt du lecteur curieux de découvrir une approche originale de textes et d'œuvres désormais canoniques.

\section{Emmanuel Guy \\ Université Paris $13-C E N E L$}

Laura Doyle and Laura Winkiel (dir.), Geomodernisms. Race, Modernism, Modernity, Bloomington, Indiana University Press, 2005, 354 p.

Cette publication, qui regroupe les contributions d'une vingtaine de chercheurs, est le fruit de différents séminaires organisés par The Modernist Studies Association Conference, de 2000 à 2003. Échanges et débats nombreux qui ont contribué, sans doute, à inscrire l'ouvrage dans une perspective de nécessaire dynamisme dans la manière de repenser les concepts et de les réagencer pour aborder la question du « modernisme » sous des angles différents.

Ce concept de « modernism », central dans l'ouvrage, donne lieu à de nombreuses mises en perspective et est le premier à passer au crible de la critique, qui choisit, tout d'abord, de ne le décliner qu'au pluriel, tant il semble en effet difficile de pouvoir postuler un seul et unique modernisme (ainsi qu'une seule et unique modernité) et tant cette notion semble impossible à circonscrire, ne cessant de se dérober et se transformant en permanence selon les époques et les points de vue.

Le lecteur, ainsi confronté d'emblée à l'impossibilité de travailler à partir d'un concept unique, découvre d'autres partis pris nécessaires :

To emplace modernism in this way - écrivent ainsi Laura Doyle et Laura Winkiel dans leur introduction - to think, rather, in terms of interconnected modernisms - requires a rethinking of periodization, genealogies, affiliations and forms. To some degree, this rethinking estranges the category of modernism itself. The term modernism breaks open, into something we call geomodernisms, which signals a locational approach to modernisms' engagement with cultural and political discourses of global modernity. (p. 3) 
Cette ligne de réflexion guide l'ensemble de l'ouvrage qui cherche à sortir des oppositions binaires si souvent réductrices (centre/périphérie; tradition/modernité...) pour essayer de penser au-delà des frontières (géographiques, conceptuelles ou textuelles) préétablies. Ce que suggère, par exemple, Susan Stanford Friedman en mettant en place son concept de «cultural parataxis » qui choisit de mettre en contact et faire résonner des textes d'époques différentes mais au sein desquels se nouent des échos très porteurs ${ }^{2}$.

Car de quelle modernité parle-t-on? De celle qui est née en Europe avec le mouvement des Lumières (et dont la langue anglaise, au travers du terme « Enlightnement» rend bien visible l'opposition qui peut se jouer avec le terme de « dark »)? De celle qui, très liée à la philosophie de Hegel, se développe dans le cadre précis de l'historicité et resurgit sporadiquement dans les discours contemporains ${ }^{3}$ ? N'existe-t-il pas, plutôt qu'une modernité, des « nœuds de modernité » qui créent des interactions des plus inattendues mais des plus motivantes intellectuellement?

Autant de questions que les diverses contributions - qui ne peuvent être que des études de cas - posent avec plus ou moins d'acuité (l'ensemble des contributions étant de qualité inégale) tout en balayant un très large champ littéraire et artistique (de la littérature au cabaret, en passant par le cinéma, du Liban aux États-Unis, de l'Inde à Cuba et à l'Afrique du Sud...).

Ce faisant, elles offrent surtout, chacune à leur manière, une façon de réorganiser des lectures, des théories et des concepts tout en forgeant des outils d'analyse souvent efficaces.

Ce travail de réagencement nous amène d'ailleurs à porter une attention particulière à la position de Fredric Jameson, citée à plusieurs reprises dans l'ouvrage, et selon laquelle « Modernity is not a concept, but rather a narrative category ${ }^{4} »$. Une conception fine, qui permet de garder au mot toute sa relativité nécessaire et lui donne une latitude d'application particulièrement large.

2. Susan Stanford Friedman, «Paranoia, Pollution, and Sexuality », dans Laura Doyle and Laura Winkiel (dir.), op. cit., p. 245-261.

3. On pense immanquablement, bien sûr, aux discours sur « l'entrée des peuples dans l'histoire » mais aussi à ceux tenus sur la capacité des peuples à se diriger eux-mêmes (en lien avec leur " capacité de modernité »). Comme le rappelle Laura Doyle avec un extrême à-propos : "Over the next century, this discourse yielded the notion that some races are born to seek freedom - and therefore deserve it - and others are not. By the later eighteenth century and until Iraq in 2004, peoples or races must, from a Western point of view, demonstrate their "capacity" for freedom, or be ruined. In the Western idea of freedom, race and modernity join hands, for the will of freedom is the very essence, according to G.W.F. Hegel and others, of "wolrd-historical", modern races. In modernity, it is above all the capacity for freedom that measures a race. »

4. Fredric Jameson, A Singular Modernity: Essay on the Ontology of the Present, New York, Verso, 2002. Cité dans les contributions de Janet Lyon (d'où est tirée la présente citation, p. 203) et Ian Baucom. 
Car le regard porté sur la (les) modernité(s) n'est-il pas, lui-même, extrêmement ancré dans un contexte spécifique et le concept même une construction d'un regard « occidental»?

Comme le rappelle très justement Janet Lyon dans son excellente contribution « Gadže Modernism », le concept de « Gypsy culture » est « a gadže construction $^{5}$ ». Ce constat peut aussi bien s'appliquer au concept de « modernism », qui, malgré toutes les différentes acceptations qu'il peut prendre au sein de la réflexion - et particulièrement dans cet ouvrage - est également le fruit d'un regard et d'une réflexion universitaire particuliers, comme le soulignait d'ailleurs finement l'introduction :

The globalization of criticism, like that of trade, inevitably sends forth the specter of appropriation, and this collection walks under its shadow. After all, this collection is in English. Only a few of us hail from universities outside the United States. The economy that supports us and these conversations (albeit less and less) is embedded in the "Western" capitalism many of these essays critique. (p. 6)

Si cela ne signifie nullement que la «modernité »n'ait pas existé ailleurs qu'en Occident (au contraire, et tout l'ouvrage le prouve), il reste cependant que le discours sur la modernité, quelles que soient les définitions que l'on donne au terme, est lui très ancré dans une réalité occidentale.

Ce constat peut, bien sûr, apparaître comme une "limite », mais si l'on considère avec Jameson que la modernité réside, pour ainsi dire, dans « l'ordre du discours ${ }^{6} »$, alors on ne peut que constater la manière dont cet ouvrage met en pratique le sujet dont il traite : outre la façon dont il nous permet de voyager au travers les lieux, les époques, les textes et les contextes, il nous offre surtout un exercice intellectuel passionnant de relectures des textes, en particuliers philosophiques. Retravaillant à partir d'Hegel, Foucault, Habermas et tant d'autres, les contributeurs nous ouvrent de nouvelles façons de penser la modernité, de manière dynamique et plutôt motivante. « Challenging », comme diraient les Américains!

Nathalie Carré

Université Paris 13 - CENEL

Modernism/Modernity, vol. 13, n 3, sept. 2006

La tendance générale de la critique littéraire actuelle est de lier l'émergence des littératures postcoloniales au pouvoir libérateur d'un autre «post»: le postmodernisme et sa fracassante remise en cause de la modernité et du « centre ». Le combat engagé par les littératures postcoloniales dans le sens d'une

5. Janet Lyon, «Gadže Modernism », dans Laura Doyle and Laura Winkiel (dir.), op. cit., p. $187-206$.

6. Et pour reprendre le titre du magistral texte de Foucault. 